\title{
RESEÑA: OBRA ESCOGIDA DE ROKEYA SAKHAWAT HOSSAIN. JORGE DIEGO SÁNCHEZ (EDITOR Y TRADUCTOR) ${ }^{1}$
}

\author{
Mónica Fernández Jiménez²
}

Jorge Diego Sánchez (Introducción, selección, edición crítica y traducción). Obra Escogida de Rokeya Sakhawat Hossain. Salamanca: Ediciones Universidad de Salamanca, 2020. ISBN: 978-84-1311-225-1.

Obra Escogida nos trae de la mano de Jorge Diego Sánchez (editor, traductor y autor de la edición crítica) y de Ediciones Universidad de Salamanca la obra nunca traducida al español de Rokeya Sakhawat Hossain (1880-1932). Rokeya, pues a esta autora se la conoce por su nombre de pila en el subcontinente surasiático, es una figura clave en los estudios de la mujer de esta región. Como indica el prólogo de Barnita Bagchi y la breve biografía incluida por Diego Sánchez, la vida y obra de Rokeya marca un punto de inflexión en la lucha por los derechos y el acceso a la educación de las mujeres y las niñas en el subcontinente indio (ha de ser recordado que la actual Partición del mismo en los estados de India, Pakistán y Bangladesh no existía en la época en la que vivió Rokeya). Su compromiso y activismo nos llega a día de hoy en la forma de sus escritos - polémicos en la época - pero también a través de la escuela que fundó primero en Bhagalpur (estado de Bihar) y más tarde en Calcuta (estado de Bengala), la Sakhawat Memorial Girls School, todavía funcionando en la actualidad. La

\footnotetext{
${ }^{1}$ Fecha de recepción: 10/04/2020.

Fecha de aceptación: 10/04/2020.

${ }^{2}$ Investigadora predoctoral en el departamento de Estudios Ingleses de la Universidad de Valladolid, España; $₫$ monica.fernandez@uva.es.
} 
edición de Diego Sánchez recoge los textos más ilustrativos de esta autora para comprender lo que supone y supuso su figura y lucha, así como una explicación de lo que el autor considera que es el acto de "ejerc[er] prácticas," definido como "la posibilidad de soñar con un mundo mejor" (16), algo "no solo posible sino también necesari[o]" (16). Y es que la obra de Rokeya se caracteriza por la utopía, definida por Anupama Mohan como la representación de un mundo ideal que a su vez mantiene el énfasis en la no existencia de dicho mundo (2012: 9). Así, Rokeya es capaz también de fijarse en los elementos que impiden no solo un cambio en la sociedad sino también un espacio de diálogo para negociarlo.

Esta edición está dividida en dos secciones; la primera recoge el trabajo crítico de Diego Sánchez titulada "Sueños, Informes y Piedras Preciosas" y la segunda ofrece la traducción de parte de su obra en el siguiente orden: "El Sueño de Sultana," una "Selección de Informes de Las Recluidas," "Fragmentos de Padmaraj o El Rubî" y "Fragmentos de 'La Caída de la Mujer"” (de Motichur I). Jorge Diego Sánchez lleva a los lectores a través de un recorrido biográfico, contextual y teórico necesario para la comprensión de la traducción que sigue. El autor destaca el carácter pionero de la propuesta de Rokeya de práctica feminista, que ha sido rearticulada por teóricas feministas cien años más tarde, así como la necesidad de estudiar su figura para comprender a autoras de origen bangladeshí más contemporáneas como son Monica Ali, Tahmima Anam o Taslina Narsin A su vez, la importancia de la escritura de Rokeya se ve reflejada en la materialización de sus distintos proyectos educativos, llevando a cabo así "distintas prácticas que unían postulados teóricos y aplicaciones participativas, reales y sistémicas" (33). Por tanto, el autor, quien considera de extrema importancia el llevar a cabo prácticas que reflejen lo textual, cree que estos textos son un valioso motor de cambio (ibid). Por último, establece la razón de su selección en tanto a que los textos 
se explican unos a otros: mientras que unos son descriptivos, otros son más reflexivos, y otros ofrecen alternativas. Y, por supuesto, se hace evidente a lo largo de la lectura de esta edición crítica que su autor aborda este trabajo con una convicción sincera y personal que se compromete para mostrar la importancia social que suponía que la obra de Rokeya fuese traducida al español por primera vez.

"El Sueño de Sultana” trata de una historia corta que refleja las realidades del sistema purdah a través de la narración en primera persona de su protagonista, Sultana, quien oníricamente (o no) viaja a una utopía llamada Tierradelasmujeres. En la historia se refleja su miedo y lo que significaba este sistema del purdah - "Me daba mucha vergüenza pensar que estaba paseando por la ciudad en mitad de la mañana, pero parecía que no había ningún hombre visible así que me relajé" (53-4) - pero también la esperanza de cambio "si los hombres que la habitan quisieran" (55). La expresión de la subjetividad en el relato muestra la absurdez del sistema, por el cual las mujeres no casadas tenían la responsabilidad de esconderse, incluso cuando alguien aparecía en su propia casa. Esta subjetividad, además, es colectiva, pues la conciencia de la protagonista se funde con la de las otras mujeres libres que conoce en Tierradelasmujeres pese a que lingüísticamente no se entiendan. Y, al final, el lector o lectora podría fácilmente observar que no todo se trata de ciencia ficción sino de una posible alegoría cuando Hermana Sara le dice a Sultana: "Cuéntales [a tus amigas] todo lo que aquí viste" (62). Imaginar una alternativa a los sistemas violentos y patriarcales que tal vez es lo que la protagonista hace - se convierte en una herramienta subversiva si se emplea colectivamente.

Antes de la selección de los trece informes extraídos de Las Recluidas se sitúa una introducción de la autora donde explica en qué consistía el sistema purdah, interesante 
para que el lector o lectora pueda entender el contexto histórico y social de Rokeya y, por lo tanto, por qué resultó en su día tan polémica y resulta en la actualidad tan adelantada a su tiempo. Tras estas anotaciones generales, es interesante que los informes se centran en la experiencia particular de una o varias mujeres, como siempre Rokeya oscilando entre lo personal y lo colectivo como parte esencial de su cometido. La descripción de las experiencias personales es crucial para plasmar el mapa emocional y psicológico que suponía el sistema para aquellas que lo sufrían a la vez que para explicarlo mejor. Por ejemplo, en el primer informe se explica cómo no solo eran los hombres los que no podían ver a estas recluidas - mujeres musulmanas no casadas sino también otras mujeres que no fuesen de su familia o trabajadoras domésticas. Ante la intrusión que describe este primer informe, las mujeres corren despavoridas de tal manera que "[b]ien se podía decir que parecía que un tigre andaba suelto por la casa por la manera en la que corrían y candaban las puertas de los dormitorios" (68). Pese a este inicio semi-humorístico, muchos de los otros informes, a través de la descripción de escenas hipotéticas particulares, se centran en lo inhumano de la sacralidad del purdah, que podía causar que mujeres fuesen lastimadas ("Informe Siete"; "Informe Dieciocho") o incluso muriesen ("Informe Ocho"; "Informe Catorce") con tal de no romper las reglas. Hacia la mitad de la selección se incluyen informes que hablan de la reclusión a otros niveles distintos a los de las zenanas - o que existen otros tipos de reclusión como el de los burkas o el de los tabúes y la vergüenza: “¿Cómo podría decirle a un hombre lo que de verdad ocurría [una mujer se había puesto de parto]? ¿No hubiese sido algo que nos avergonzaría públicamente delante de él?” (77).

Como “El Sueño de Sultana," los fragmentos de Padmaraj o El Rubí, la siguiente obra de la que Diego Sánchez traduce una sección, presentan una suerte de utopía, esta mucho menos fantasiosa y bastante más factible por el motivo de que la institución 
descrita en sus páginas se sitúa un mundo que parece ser el contemporáneo de Rokeya y también porque se asemeja a la propia institución fundada por esta en 1915: la Asociación de Mujeres Bengalíes y Musulmanas (Anjuman-e-Khawatin-e-Islam). Se trata de Tarini Bhavan, un hogar fundado por la viuda Dina-Tarini para dar cobijo a las viudas y a las mujeres desamparadas, así como para educar a las niñas. Este lugar es utópico no solo en la práctica que lleva a cabo sino también en la coexistencia que se da en el mismo de distintas religiones que conviven en igualdad y sin violencia ni supremacía. Tarini Bhavan también tiene lugar fuera de la influencia gubernamental india o colonial británica. Es decir, como dicta la utopía al estilo Rokeya que Diego Sánchez explica en la introducción, la narrativa da la vuelta a la sociedad (subvierte lo que se describe en los Informes) para a su vez resaltar el existente potencial femenino que estos sistemas apagan y recluyen (16). Asimismo, esta narración resulta rompedora ya que toca algunos temas extremadamente contemporáneos que convierten a Rokeya en una adelantada a su tiempo: la necesidad de que el poder hegemónico esté alejado de la gestión de estas instituciones, la necesidad de una educación libre sin intromisión gubernamental o financiera (las donaciones debían ser secretas), el rechazo a la ostentación y la necesidad de la sororidad así como de la autonomía de las mujeres que en Tarini Bhavan trabajan para sí mismas a no ser que fuesen enfermas o minusválidas. A través de los diálogos, los personajes establecen que los ritos de la sociedad patriarcal - lo que da prestigio - no definen la respetabilidad real ni mucho menos, ya que están corruptos.

El último texto traducido, una serie de capítulos de "La Caída de la Mujer," uno de los escritos que se recogieron en Motichur I, supone una buena conclusión que engloba a los textos anteriores. El texto, en forma de diálogo socrático (como explica Diego Sánchez en la introducción), trata del ancestral sometimiento de todas las mujeres 
y su perpetuación a través de sistemas religiosos, políticos y sociales. El texto enfatiza que esta situación se perpetúa porque no existe posibilidad de agencia para las mujeres, quienes se mantienen en su situación a través de una serie de chantajes emocionales derivados de su falta de acceso a la educación y a la participación ciudadana. La conexión entre la educación y la emancipación como indica Bachi en el prólogo (9) se hace evidente a lo largo de esta selección que resume de manera excelente la significancia de Rokeya: como se ha mencionado ya varias veces en esta reseña, el texto tiene vida más allá de su lectura ya que interpela a las mujeres para que desparezca lo que hay de su propio consentimiento, propone alternativas y señala culpables sistémicos.

\section{Referencias bibliográficas}

Mohan, Anupama. 2012. Utopia and the Village in South Asian Literatures. London: Palgrave McMillan. 